\title{
A symbolic projection of Langton's Ant (Extended Abstract)
}

\author{
Anahí Gajardo ${ }^{\dagger}$ \\ GI ${ }^{2} M A$, Departamento de Ingeniería Matemática, Universidad de Concepción, \\ Casilla 160-C, correo 3, Concepción, Chile
}

The Langton's ant is studied from the point of view of topological dynamical systems. A new approach which associate a subshift to the system is proposed. The transition rule is generalized to the family of bi-regular graphs $\Gamma(k, d)$ and the dependence of the dynamical system on $k$ and $d$ is analyzed. A classification of the $\Gamma(k, d)$ graphs based on the dynamical properties of the subshift is established. Also a hierarchy is defined on the graphs through the subset relation of the respective subshifts. The analysis are worked out by establishing an algebraic characterization of the forbidden words of the subshift.

Keywords: Symbolic Dynamics, Lorentz Lattice Gas, Cayley Graphs

\section{Introduction}

Langton's Ant is a very simple discrete dynamical system introduced by Cris Langton [Lan86] as an example of systems presenting life-like behaviors. Its celebrity comes from an unproved conjecture, which remains open from more than 10 years: In all the computer-simulations, when at the beginning there are some cells in black color and the grid is big enough, the ant eventually falls in a periodic movement with drift, leaving behind a periodic pattern. The periodic movement is the same in every case (see Figure 1). But nobody knows if that happens for any initial confi guration with a fi nite number of black cells or not. In this article, we consider a generalization of the system to arbitrary planar graphs. This generalization is simple and independent on the graph in the sense that the ant do not need to know any information about the graph except for its planar projection. The same generalization has been already studied over the regular grids [KC91, GBCB99, Wan95]. Different behaviors appear over the three regular grids. We try to characterize the relation between the ant behavior and the graph topology. We consider the class of bi-regular graphs $\Gamma(k, d)$. We take a dynamical system approach classifying the graphs with respect to properties as transitivity and sofi city of the system. Similar systems have already been studied in the context of dynamical systems [BT92, BT93, GBCB99, Qua99]. The new feature of the present work is that we are not concentrating on the ant's dynamical system it self but on a particular partition subshift of it. The partition is defi ned in such a way that the partition subshift contains all the relevant information of the system (in particular the ant's motion). Due to this, the properties of the subshift reflects in a very

${ }^{\dagger}$ This work was supported by the Direrección de Investigación of the Universidad de Concepción, Chile. 1365-8050 (c) 2003 Discrete Mathematics and Theoretical Computer Science (DMTCS), Nancy, France 

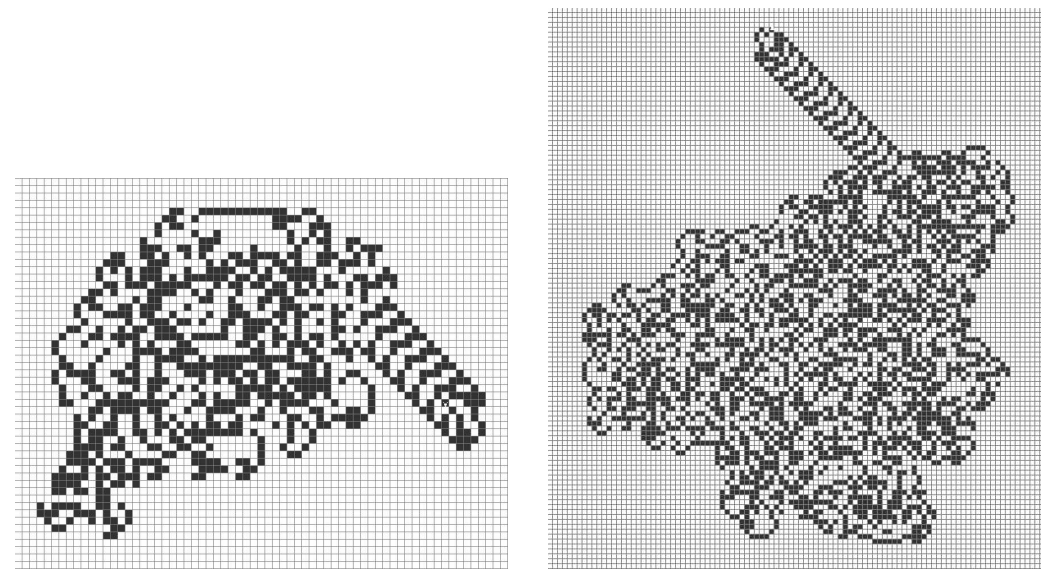

Fig. 1: The configuration at times 10700 (left) and 47500 (right) when starting from two different initial configurations.

sensible way the properties of the original system. In this paper, we study and characterize this subshift. Several results presented here are consequences of previous results of the authors [GMG01b, Gaj01]. In Section 2, the generalization of the ant to planar graphs is presented. In Section 3, we defi ne the subshift associated to the system and we show how its forbidden words are related to the null words of a group. Section 4 establishes several strong properties of the subshift associated to the $\Gamma(k, d)$ graphs where $d \geq 5$. In particular its language is characterized through a simple congruence. Finally, in Sections 5 and 6 we show the properties of the different subshifts associated to each $\Gamma(k, d)$ graph.

\section{Langton's Ant on planar graphs}

Given a graph represented on the plane in such a way that its edges do not intersect each other, the ant's system is defi ned as follows:

- Each vertex (preferably called cell) has an associated state which can be L or R.

- The ant is represented by an arrow laying over a graph edge, heading to a cell, say $h$.

- At each iteration:

- The ant moves to one of the edges adjacent to $h$. It moves to the closer edge to the left of $h$ if $h$ is in state $\mathrm{L}$ and it moves to the closer edge to the right otherwise.

- $h$ changes of state.

The system dynamics may be different for different representations of the same graph. When referring to a graph, a particular representation is considered implicitly. In this article we focus principally on a particular class of planar graphs: the $\Gamma(k, d)$ graphs. The graph $\Gamma(k, d)$ is the locally fi nite planar graph whose cells have $d$ neighbors and whose faces have $k$ sides. $\Gamma(k, d)$ is fi nite for $k=3$ and $d<6, k=4$ or $k=5$ and $d<4$, or when $k=6$ and $d<3$. Finite $\Gamma(k, d)$ correspond to the six platonic regular polygons. 


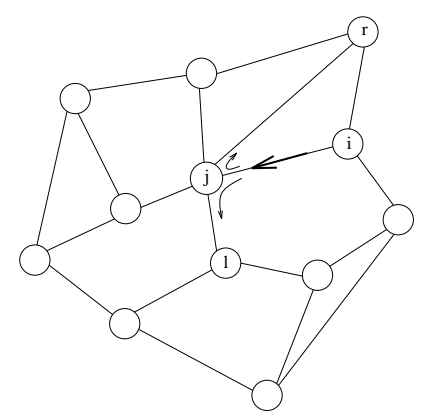

Fig. 2: Depending on the cell's state, the ant will turn to the left or to the right.

Only $\Gamma(6,3), \Gamma(4,4)$ and $\Gamma(3,6)$ can be embedded in $\mathbb{R}^{2}$ with edges of constant lengths; they correspond to the hexagonal, square and triangular grids respectively. The rest of the cases corresponds to the so called "hyperbolic graphs", and can be embedded in the hyperbolic plane. Figure 3 shows a part of the $\Gamma(7,3)$ graph. For these graphs, the planar representation is given together with the graph defi nition and

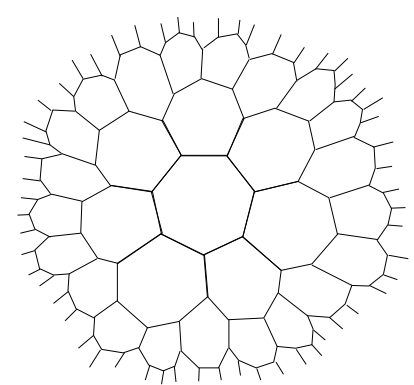

Fig. 3: The $\Gamma(7,3)$ graph.

it is unique up to homeomorphism.

\section{Symbolic representation}

In this paper we study the ant with the perspective of topological dynamical system. A topological dynamical system is a topological space $X$ called "phase space", together with a continuous function $f: X \rightarrow X$. There are several ways to formalize the ant as a dynamical system depending on the choice of the representation of the system state. This problematic is discussed by Kůrka in [K97] for the case of Turing machines. Kurka shows that different representations of the system have different properties. In a fi rst approach, the system state can be assumed as a confi guration $c: V \rightarrow\{\mathrm{L}, \mathrm{R}\}$, together with the ant position. Then, the set of states is $A_{G}=\{\mathrm{L}, \mathrm{R}\}^{V} \times \vartheta_{G}$. Where $\vartheta_{G}$ represents the set of pairs $(i, j) \in V \times V$, such that $\{i, j\} \in E$. With the product topology, $A_{G}$ is a non-compact set. The transition function $f$ acts over $c(j)$ and $(i, j)$. It is continuous, one-to-one and onto. This representation is the more natural one, and it is the best for simulation. Nevertheless, it is not adequate for the analysis of the ant's movement. 
The state representation contains too much information, because the cells that are never visited by the ant, are not interesting and shifting a confi guration, together with the ant position does not change the ant's movement. We defi ne another dynamical system that is related with $\left(A_{G}, f\right)$ through the projection function $\pi: A_{G} \rightarrow\{L, R\}$ where $\pi(c,(i, j))=c(j)$. The sequence $\left(\pi\left(f^{n}(x)\right)\right)_{n \in \mathbf{N}}$ contains the states of all the cells that the ant will visit when the starting state is $x$. At the same time, it is itself a precise description of the ant's trajectory. If we know the starting ant's position and $\left(\pi\left(f^{n}(x)\right)\right)_{n \in \mathbf{N}}$, it is possible to infer the whole ant's trajectory and we can determine its regularities if there are some. Defi ning the set:

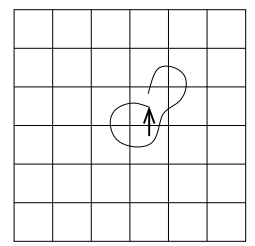

$\mathrm{x}=$ LLLLRLLLL $\ldots$.

Fig. 4: The sequence generated by the configuration where all the cells are in L state.

$$
H_{G}=\left\{\left(\pi\left(f^{n}(x)\right)\right)_{n \in \mathbf{N}} \in\{L, R\}^{\mathbf{N}} \mid x \in A_{G}\right\}
$$

and the shift function $\sigma: H_{G} \rightarrow H_{G}$ by $\sigma\left(\left(a_{0}, a_{1}, a_{2}, \ldots\right)\right)=\left(a_{1}, a_{2}, a_{3}, \ldots\right)$, we have the following scheme:

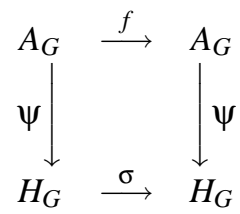

With $\psi(x)=\left(\pi\left(f^{n}(x)\right)\right)_{n \in \mathbf{N}}$. It is easy to see that $H_{G}$ is closed on $\{L, R\}^{\mathbf{N}}$ and that $\psi$ is onto and continuous. $H_{G}$ is a subshift, i. e., it is invariant under the function $\sigma$. In spite of $\psi$ not being one-to-one, $\psi(x)$ contains all the information that is relevant in the ant's dynamics. In some sense, two states $x$ and $x^{\prime}$ are equivalent when $\psi(x)=\psi\left(x^{\prime}\right)$. We think that the properties of $\left(H_{G}, \sigma\right)$ reflect the complexity of $\left(A_{G}, f\right)$ even if they do not coincide, in general, with the properties of $\left(A_{G}, f\right)$. On the other hand, $\left(H_{G}, \sigma\right)$ is a simple and homogeneous representation of $\left(A_{G}, f\right)$, in the sense that $H_{G} \subset\{L, R\}^{\mathbf{N}}$ for any $G$, then we work within a single space, we apply the same tools and describe the different properties in the same context for any graph. The set $H_{G}$ depends on $G=(V, E)$. For example, if the faces of $G$ have at most $r$ sides, then the word $L^{r+1}$ is forbidden in $H_{G}$.

\subsection{Basic definitions}

In the following we will assume that the underlying graph, $G$, is a $\Gamma(k, d)$ graph. In order to simplify the notation we will denote $A_{k d}=A_{\Gamma(k, d)}$ and $H_{k d}=H_{\Gamma(k, d)}$. Also we denote the cell, edge and arc sets of $\Gamma(k, d)$ by $V_{k d}, E_{k d}$ and $\vartheta_{k d}$, respectively. The function $f$ defi nes a relation between the arcs in $\vartheta_{k d}$ by $(i, j) \propto\left(i^{\prime}, j^{\prime}\right) \Leftrightarrow \exists c, c^{\prime} \in\{R, L\}^{V_{k d}}$ such that $f(c,(i, j))=\left(c^{\prime},\left(i^{\prime}, j^{\prime}\right)\right)$. In other words, $(i, j) \propto\left(i^{\prime}, j^{\prime}\right)$ if $\left(i^{\prime}, j^{\prime}\right)$ is the arc to the right or to the left of $(i, j)$. Let us call $T_{k d}$ the graph associated to this relation. A curious property is that when $d$ is even, $T_{k d}$ has two connected components. This is because when $d$ is 
even, no edge can be visited in the two orientation during a single trajectory (see [Gaj01] Ch.3.1). As we will see, $T_{k d}$ has a group structure.

Definition 1 Given two integers $k$ and $d$, we define the group $D_{k d}$ as follows ${ }^{\ddagger}$. If $d$ is even:

$$
D_{k d}=\left\langle R, L \mid R^{k}, L^{k},\left(R L^{k-1}\right)^{d / 2},\left(L R^{k-1}\right)^{d / 2}\right\rangle
$$

If $d$ is odd:

$$
D_{k d}=\left\langle R, L \mid R^{k}, L^{k},\left(R L^{k-1}\right)^{d},\left(L R^{k-1}\right)^{d},\left(\left(R L^{k-1}\right)^{\lfloor d / 2\rfloor} R\right)^{2},\left(\left(L R^{k-1}\right)^{\lfloor d / 2\rfloor} L\right)^{2}\right\rangle
$$

Lemma 1 If $d$ is even, then $T_{k d}$ has two connected components, each of them being isomorphic to the Cayley graph ${ }^{\S}$ of $D_{k d}$

If $d$ is odd, $T_{k d}$ is isomorphic to the Cayley graph of $D_{k d}$.

Since $\Gamma(k, d)$ has not, in general, an algebraic structure w(see [Cha95]), we will prefer to use $D_{k d}$ to refer to the cells and arcs of $\Gamma(k, d)$. First, from Lemma 1 the arcs of $\Gamma(k, d)$ that are potentially reached by the ant are exactly the elements of the group $D_{k d}$. We identify the $\operatorname{arcs}$ of $\Gamma(k, d)$ with the words in $\{L, R\}^{*}$ modulo $D_{k d}$. In particular the empty word, $e$, will denote a fi xed arc, say $\left(a_{-1}, a_{0}\right)$. Second, a cell of $\Gamma(k, d)$ is uniquely determined by an arc that exits from it. Then, given a word $\left(w_{i}\right)_{i=0}^{n} \in\{R, L\}^{*}$ it defi nes uniquely a sequence $a=\left(a_{i}\right)_{i=-1}^{n+1} \in V_{k d}$ such that $\left(a_{i}, a_{i+1}\right)=w_{0} . . w_{i}$ for all $i \in\{-1, . ., n\}$. We say that the sequence $a$ is the sequence of cells visited by the path $w$ and we defi ne $\varphi:\{R, L\}^{*} \rightarrow V_{k d}^{*}$ by $\varphi(w)=a$. Since an arc determines uniquely the cell from which it exits, we could refer the cells through a word of $D_{k d}$ too. Several arcs exit from the same cell and this can be characterized algebraically: Two arcs $s$ and $t$ in $D_{k d}$ exit from the same cell if and only if there exists $i \leq d$ such that $s\left(R^{k-1} L\right)^{i}=t$ modulo $D_{k d}$.

Also we can defi ne the states that these cells need to have in order to make the ant to actually follow the path $w$.

$$
c\left(a_{i}\right)=w_{\min }\left\{j \mid a_{j}=a_{i}\right\} \quad 0 \leq i \leq n
$$

The rest of the cells can be in any state, then we arbitrarily assign $c(b)=L$ for all $b \notin\left\{a_{i}\right\}_{i=0}^{n}$. We defi ne $\phi:\{R, L\}^{*} \rightarrow\{L, R\}^{V_{k d}}$ by $\phi(w)=c$. Both functions $\phi$ and $\varphi$ are well defi ned for every $w \in\{R, L\}^{*}$ and they can be extended to infi nite sequences $w \in\{R, L\}^{\mathrm{N}}$ by continuity. By defining $\bar{R}=L$ and $\bar{L}=R$ we can rewrite the function $f$ as follows.

$$
f(c, w)=\left(c^{\prime}, w c\left(\varphi(w)_{|w|}\right)\right), \text { with } c^{\prime}\left(\varphi(w)_{|w|}\right)=\overline{c\left(\varphi(w)_{|w|}\right)}
$$

A word $w=w_{0} . . w_{n} \in\{R, L\}^{*}$ is called an ant's word if $w \in L\left(H_{k d}\right)$. A word $w=w_{0} . . w_{n} \in\{R, L\}^{*}$ is an ant's word if and only if $\psi(\phi(w), e)_{0} . . \psi(\phi(w), e)_{n}=w$. From this, we can determine a basis of forbidden words of $H_{k d}$, i. e., a set, $F_{k d}$ of words such that a word $w$ is in $H_{k d}$ if and only if $w$ has no factor in $F_{k d}$.

$$
F_{k d}=\left\{w_{0} \ldots w_{n} \in\{R, L\}^{*} \mid \varphi(w)_{0}=\varphi(w)_{n} \wedge \varphi(w)_{0} \notin\left\{\varphi(w)_{i}\right\}_{i=1}^{n-1} \wedge w_{0}=w_{n}\right\}
$$

The condition $\varphi(w)_{0}=\varphi(w)_{n}$ says that the ant points to the same cell at times 0 and $n$. The condition $\varphi(w)_{0} \notin\left\{\varphi(w)_{i}\right\}_{i=1}^{n-1}$ says that this cell is not visited between times 1 and $n-1$. Finally, condition $w_{0}=w_{n}$

\footnotetext{
$\$$ In the notation $\left\langle R, L \mid \alpha_{1}, \alpha_{2}, . ., \alpha_{n}\right\rangle, R$ and $L$ are the set of generators, and $\left\{\alpha_{i}\right\}_{i=1}^{n}$ is the set of relators which defi nes the null words of the group (see [LS77]).

$\S$ Cayley graphs has been introduced by Cayley in [Cay78]
} 
says that the ant turns to the same direction at this cell in both times 0 and $n$, which contradicts the ant's rule. It will be useful to know which words corresponds to simple paths. We say that a word $w=w_{0} . . w_{n}$ is simple if $\varphi(w)_{i} \neq \varphi(w)_{j}$ for every $-1 \leq i<j \leq n+1$. We denote by $S_{k d}$ the set of simple words. $S_{k d}$ is a factorial language but it is not extensible in general. All the simple words are ant's words, i. e., $S_{k d} \subset L\left(H_{k d}\right)$. We say that a word $w=w_{0} . . w_{n}$ is a simple cycle if $w_{0} . . w_{n-1}$ is simple and $\varphi(w)_{-1}=\varphi(w)_{n+1}$. With these defi nitions we are ready to study $H_{k d}$.

\section{When $d \geq 5$}

In this section we assume that $d \geq 5$ if $k \geq 4$ and that $d \geq 6$ if $k=3$. This hypothesis allows us to prove the following lemma. The proof uses relations between the number of nodes, edges and faces enclosed by the cycle and the Euler theorem.

Lemma 2 1. If $w=w_{0} . . w_{n}$ is a simple cycle, then $w=R^{k-1}$ or $w=L^{k-1}$.

2. If $u=u_{0} . . u_{n}$ and $v=v_{0} . . v_{m}$ are simple, then $\varphi(u)_{n+1} \neq \varphi(v)_{m+1}$.

Corollary $1 S_{k d}$ is the language obtained by forbidding the factors $R^{k-1}$ and $L^{k-1}$.

In the present case, $S_{k d}$ do not depends on $d$, then we will call it $S_{k}$. Lemma 2 implies that the subgraph generated by $S_{k}$ in $D_{k d}$ is a tree. It also implies that the graph induced by the arcs of $S_{k}$ in $\Gamma(k, d)$ is also a tree. The projection of $S_{4}$ in $\Gamma(4,5)$ is depicted in Figure 5(a) and the graph generated by $\varphi\left(S_{4}\right)$ in $\Gamma(4,5)$ is depicted in Figure 5(b). Figure 5(a)shows the set of cells that the ant can visit through a simple path. In

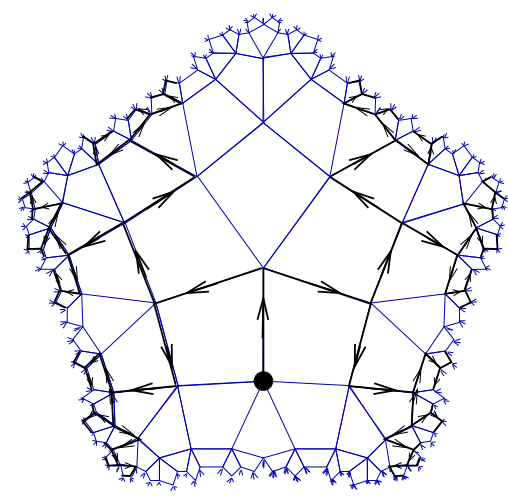

(a)

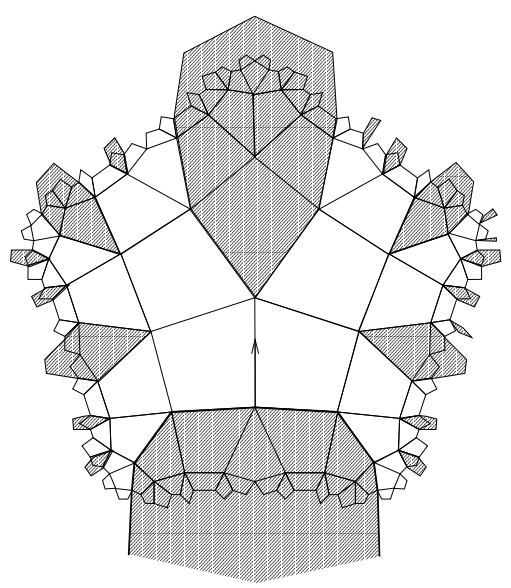

(b)

Fig. 5: (a) The graph generated by $S_{k}$ in $\Gamma(4,5)$. (b) The graph generated by $\varphi\left(S_{k}\right)$ in $\Gamma(4,5)$ is composed by the cells and edges inside the white zone or over its boundary.

[Gaj01] we proved the following theorem.

Theorem 1 If in some iteration the ant is between two cells in the same state, then its trajectory after time $t$ does not exits from a subgraph that has the shape of the tree of faces depicted in Figure 5(b). 
In this paper, we show a new proof of this theorem based on the present algebraic formulation. The gain is a more precise understanding of the system given by three lemmas and one additional theorem that we develop in the following. In the present terms, we describe the cell states through a word $w=w_{0} . . w_{n}$. This assumes that the ant is pointing to the cell $w_{0}$ at time 0 . At time 1 the ant is between the cells $w_{0}$ and $w_{1}$ and the state of the cell $w_{0}$ has changed. Then, the hypothesis of the Theorem 1 translates as $w_{0} \neq w_{1}$, and the thesis refers to the word $w_{1} . . w_{n}$ which is the ant's path after time 1 . Theorem 1 can be stated in the present terms as:

$$
\text { If } w=w_{0} . . w_{n} \in L\left(H_{k d}\right) \text { and } w_{0} \neq w_{1} \text { then } \varphi\left(w_{1} . . w_{n}\right) \subset \varphi\left(S_{k}\right) .
$$

It will be useful to consider the congruence $C_{k}=\left\langle R, L \mid R^{k}=e, L^{k}=e\right\rangle$, i. e., the quotient of the free monoid $\{L, R\}^{*}$ by the relations $R^{k}=e, L^{k}=e$. Congruences are different from groups because in the congruences the inverse of the generators are not symbols of the alphabet. $C_{k}$ has two important properties. First, every word in $\{L, R\}^{*}$, has a unique minimal representation in $C_{k}$. Second, it is always possible to obtain this minimal representation by making monotonic reductions. A congruence with these two properties is called confluent (from the nomenclature of Berstel [Ber77]). Since the relators that defi ne $G_{k}$ are of the form " $e$ is equal to something", $C_{k}$ is what Cochet and Nivat $[\mathrm{CN}]$ call a trivial congruence (it is also a basic congruence from the Berstel nomenclature). This implies several properties, in particular, all the congruence classes are algebraic languages. We rewrite one of their results for the particular case of congruence $C_{k}$. Let us recall that $w$ is a prime word if it reduces to $e$ and it has no any proper left factor which reduces to $e$.

Property 1 (Cochet \& Nivat) If $w$ is prime, then whether $w \rightarrow^{*} R^{k}$ or $w \rightarrow^{*} L^{k}$ but not both.

Lemma 2 implies that every cycle contains the sequence $R^{k}$ or $L^{k}$. Of course, no word in $S_{k}$ reduces to $e$ in $C_{k}$. We will prove that the relations $R^{k}=e$ and $L^{k}=e$ are the unique relations needed to reduce an ant's word in $D_{k d}$. This is equivalent to prove that $\varphi(w) \subset \varphi\left(S_{k}\right)$ for every ant's word $w$. If we prove that for all $w \in L\left(H_{k d}\right)$ the minimal representation of $w$ in $C_{k}$ is in $S_{k}$ we are done. But this is not true, in fact, the word $R^{k-1}$ is in $L\left(H_{k d}\right)$ and it is minimal in $C_{k}$ but it does not belong to $S_{k}$. However $\varphi\left(R^{k-1}\right) \subset \varphi\left(S_{k}\right)$, because $\varphi\left(R^{k-1}\right)_{k-1}=\varphi\left(R^{k-1}\right)_{-1}$. In general, if $v=v_{0} . . v_{n} \in S_{k}$, either $v R \in S_{k}$ or $v R$ has $R^{k-1}$ as right factor, in which case $\varphi(v R)_{n+2}=\varphi(v R)_{n+2-k} \in \varphi\left(S_{k}\right)$. Then it is enough to prove that for all $w \in L\left(H_{k d}\right)$ the minimal representation of $w$ in $C_{k}$ is a word in $S_{k}$ concatenated with the symbol $R$ or $L$, i. e., it is in $S_{k}(R \cup L)$. In the following lemmas we will work under the assumption that $w$ satisfi es $\varphi(w) \subset \varphi\left(S_{k}\right)$. This implies several strong properties. To conclude we will consider the shortest word that do not satisfi es the assumption and we will contradict by applying the lemmas to its factors. Let us consider the following set of words.

$$
\begin{aligned}
I_{k}= & \left\{\overline{w_{n}} w_{0} w_{1} . . w_{n-1} \overline{w_{n}} \mid w_{0} . . w_{n} \text { is a prime word in } C_{k}\right\} \\
& \cup\left\{w_{0} w_{1} . . w_{n-1} w_{n} w_{0} \mid w_{0} . . w_{n} \text { is a prime word in } C_{k} \text { and } w_{1} . . w_{n} \text { do not have any left null factor }\right\}
\end{aligned}
$$

Remark 1 If $\varphi(w) \subset \varphi\left(S_{k}\right)$, we have that $I_{k} \subset F_{k d}$. This can be verified by observing that if $w_{0} . . w_{n}$ reduces to e then $\varphi\left(w_{0} \ldots w_{n} w_{0}\right)_{0}=\varphi\left(w_{0} \ldots w_{n} w_{0}\right)_{n+1}$ and $\varphi\left(\overline{w_{n}} w_{0} \ldots \overline{w_{n}}\right)_{0}=\varphi\left(\overline{w_{n}} w_{0} \ldots \overline{w_{n}}\right)_{n+1}$. The fact that $w_{0} . . w_{n}$ is prime and that $\varphi(w) \subset \varphi\left(S_{k}\right)$ assures that the cell $\varphi(w)_{0}$ is never visited between times 1 and $n$. See Figure 6 to visualize this. 

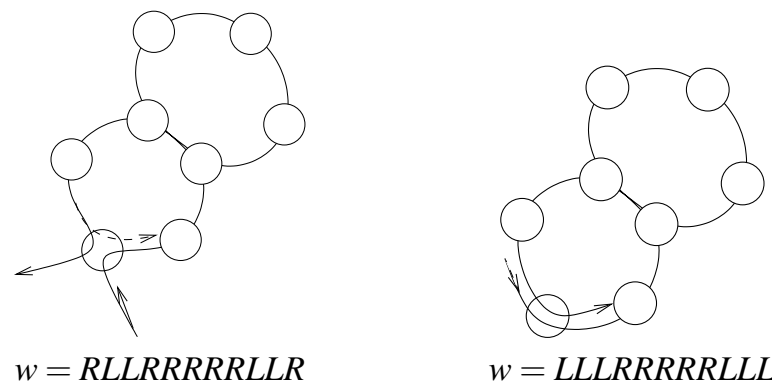

Fig. 6: Two exemples of words in $I_{k}$

Lemma 3 If $w \in L\left(H_{k d}\right)$ is a prime word in $C_{k}$ that satisfies that for any $i \in\{0, . ., n\}$ the minimal representation, $u_{i}$, of $w_{0} . . w_{i}$ in $C_{k}$ is in $S_{k}(R \cup L)$, then $w$ has a unique factorization

$$
w=x v_{1} x v_{2} \ldots x v_{k-2} x x
$$

where $x \in\{R, L\}$ and for all $j, v_{j}$ is a prime word that satisfies:

1. $v_{j} \rightarrow^{*} \bar{x}^{k}$,

2. if $v_{j}=\bar{x} g_{1} \bar{x} g_{2} \cdot . \bar{x} g_{k-1} \bar{x}$, then $g_{1}=g_{k-1}=e$ and

3. $v_{j}$ do not have $\bar{x} x$ as left factor nor $x \bar{x}$ as right factor.

Lemma 3 talks about the shape of $\varphi(w)$ and says that a closed ant's path can be decomposed in a series of closed paths that do not intersects between them and that are visited only one time each by the ant. Its proof is based on a factorization theorem by Cochet and Nivat $[\mathrm{CN}]$ and Remark 1. The next lemma talks about the states of the cells after the passage of the ant.

Lemma 4 If $w=w_{0} . . w_{n} \in L\left(H_{k d}\right)$ is a prime word in $C_{k}$ that satisfies that for any $i \in\{0, . ., n\}$ the minimal representation, $u_{i}$, of $w_{0} . . w_{i}$ in $C_{k}$ is in $S_{k}(R \cup L)$, then for a given cell $\varphi(w)_{j}$ we know that:

1. if there is some $i$, such that $\varphi(w)_{i}=\varphi(w)_{j}$ and $u_{i-1}$ has $R^{k-1}$ (or $\left.L^{k-1}\right)$ as right factor then $\varphi(w)_{j}$ has been visited by the ant two or three times where $i$ was the last one, then its state at time $n$ is $L$;

2. otherwise $\varphi(w)_{j}$ have been visited one or two times and the last time it state was $\left(u_{i}\right)_{\left|u_{i}\right|}$ then its state at time $n$ is $\overline{\left(u_{i}\right)_{\left|u_{i}\right|}}$.

Remark 2 Every cycle in $C_{k}$ contains a vertex which has $R^{k-1}$ or $L^{k-1}$ as right factor. This implies that if the ant enters $\varphi(w)$ after time $n$, it will not make any closed path before exiting $\varphi(w)$. With a little more work it can be proved that if the ant exits from $\varphi(w)$, then it follows a path LR or RL which would be at the beginning of a cycle if we are assuming that the ant will comes back to $a-1$. This is not possible from Lemma 3. This implies that the ant cannot come back to $a_{-1}$ from the right if it has already followed a cycle on the right hand. This gives a bound on the number of times that the ant can visit a given cell. The proof of the next lemma is based on this idea. 
Lemma 5 If $w \in L\left(H_{k d}\right)$ and for any pair $i<j u_{i j}$ is the minimal representation in $C_{k}$ of $w_{i} . . w_{j}$, then $u_{i j} \notin\left\{R L^{k-1} R, L R^{k-1} L\right\}$ for all $i, j$.

From this lemma we conclude Theorem 1. We remark from Lemma 5 that only the relators $R^{k}$ and $L^{k}$ can be used to reduce a word in $D_{k d}$ from this and Remark 2 we conclude the following theorem.

Theorem 2 If $d \geq 5$ and $k \geq 4$, or $d \geq 6$ and $k=3$, then for all $w \in L\left(H_{k d}\right)$ it holds that:

1. $F_{k d}=I_{k}$ and

2. every cell is visited 4 times or less.

\section{Inclusions}

In Section 5 we saw that $H_{k d}$ is constant on $d$ when $d \geq 5$. Moreover, since $R^{k+1} \notin L\left(H_{k 5}\right)$ we have that every word in $L\left(H_{k 5}\right)$ is simple in $\Gamma\left(k^{\prime}, 5\right)$ for all $k^{\prime} \geq k+2$, then $L\left(H_{k 5}\right) \subset L\left(H_{k^{\prime} 5}\right)$ for all $k^{\prime} \geq k+2$. Several inclusions like this can be proved by using the same techniques used to prove Lemma 2.

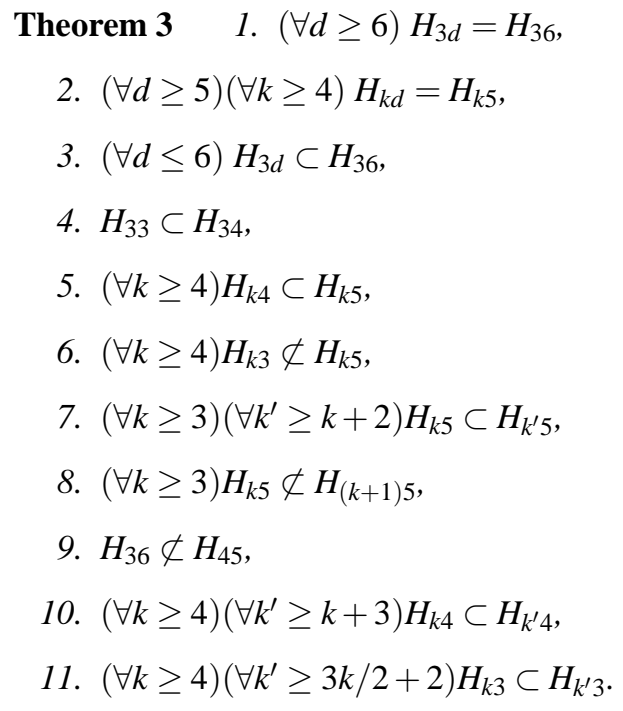

\section{Properties}

We have studied the properties of $\left(H_{\Gamma(k, d)}, \sigma\right)$ and their dependence on $k$ and $d$. They are listed in Table 1. We assume that $k$ and $d$ are such that $\Gamma(k, d)$ is an infi nite graph. A subshift is always positively expansive so this property is true for any $k$ and $d$. Transitivity is related with the existence of periodic points in $A_{k d}$, if there are periodic points in $A_{k d}$ then $H_{k d}$ is not transitive. It can be shown that for every $k, A_{k 3}$ has a periodic point. The sofi city is related with the length of the closed ant's paths. In general any length is possible, this implies that the system has infi nite memory effects, then it cannot be sofi c. $H_{5}$ is synchronizing for every $k \geq 4$, this is a consequence of Lemma 3, the synchronizing words are $L R L R$ and $R L R L$. Finally, $H_{36}$ is of fi nite type. This is because there are only two closed ant's paths in $\Gamma\left(H_{36}\right)$. Then the length of the closed ant's paths is bounded. Figure 7 shows the automaton that recognizes $L\left(H_{36}\right) . H_{36}$ has period two. 


\begin{tabular}{|c|c|c|c|c|c|c|}
\hline Transitive & Mixing & Positive Entropy & \multicolumn{2}{|l|}{ Synchronizing } & not Sofic c & SFT \\
\hline$d \geq 4$ & $d \geq 4$ & always & $d \geq 5$ & $d \geq 6$ & & \\
& $k \geq 4$ & & $k \geq 4$ & $k \geq 3$ & $k \geq 4$ & $k=3$ \\
\hline
\end{tabular}

Tab. 1: The dynamical properties of $H_{k d}$.

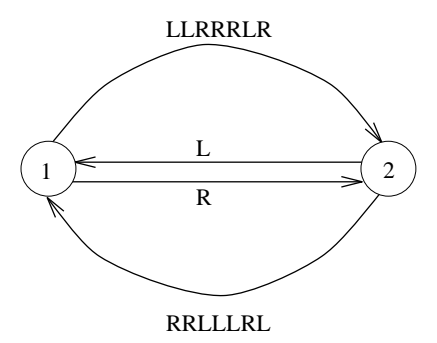

Fig. 7: The automaton that recognizes $L\left(H_{36}\right)$.

\section{References}

[Ban71] E. R. Banks. Information Processing and Transmission in cellular automata. PhD thesis, Mass. Inst. of Tech., Cambridge, Mass., 1971.

[BCG82] E. Berlekamp, J. H. Conway, and R. Guy. Winning Ways for Your Mathematical Plays, volume 2. Academic Press., 1982.

[Ber77] J. Berstel. Congruences plus que parfaites et languages alg'ebriques. \$minaire d'Informatique Théorique, Institut de Programmation, pages pp 123-147, 1976-1977.

[BT92] L. A. Bunimovich and S. Troubetzkoy. Recurrence properties of lorentz lattice gas cellular automata. J. of Stat. Physics, 67:289-302, 1992.

[BT93] L. A. Bunimovich and S. Troubetzkoy. Topological dynamics of flipping lorentz lattice gas models. J. of Stat. Physics, 72:297-307, 1993.

[BT94] L. A. Bunimovich and S. Troubetzkoy. Rotators, periodicity, and absence of diffusion in cyclic cellular automata. J. of Stat. Physics, 74:1-10, 1994.

[BT97] O. Beuret and M. Tomassini. Behaviour of multiple generalized langton's ants. In Artificial Life $V$, pages 45-50. 1997.

[Bun96] L. A. Bunimovich. Many-dimensional lorentz cellular automata and turing machines. Int. Jour. Bif. Chaos, 6:1127-1135, 1996.

[Bun00] L. A. Bunimovich. Walks in rigid enviroments. Physica A, 279, 2000.

[Cay78] A. Cayley. The theory of groups: graphical representations. Amer. J. Math, 1:174-176, 1878.

[Cha95] T. Chaboud. Pavages et Graphes de Cayley Planaires. PhD thesis, E. N. S. Lyon, 1995. 
[Cho99] B. Chopard. Complexity and cellular automata models. In S. Ciliberto, T. Dauxois, and M. Droz, editors, Physics of Complexity. Editions Frontières, Gif-sur-Yvette, 1999.

[CL90] J. H. Conway and J. C. Lagarias. Tiling with polyominoes and combinatorial group theory. Journal of Combinatorial Theory, A53:183-208, 1990.

[CN] Y. Cochet and M. Nivat. Une generalization des ensembles de dyck. Israel J. Mathematics.

[Coh92] E. G. D. Cohen. New types of diffusion in lattice gas cellular automata. In M. Mareschal and B. Holian, editors, Microscopic Simulations of Complex Hydrodynamic Phenomena. Plenum Press, 1992.

[Con72] J. H. Conway. Unpredictable iterations. In Number Theory Conference, pages 49-52, Boulder, Colorado, 1972. University of Colorado.

[Dev98] R. Devaney. Introduction to Chaotic Dynamical Systems. Addison Wesley Pub. Comp., 198.

[Dew89] A. K. Dewdney. Computer recreations: Two-dimensional turing machines and tur-mites make tracks on a plane. Scientific American, pages 124-127, September 1989.

[Dew90] A. K. Dewdney. Computer recreations:. Scientific American, pages 118-121, March 1990.

[Gaj98] A. Gajardo. Aut'omatas celulares universales en 2 dimensiones, con vecindades de tamaño 3 y 4. engeneering thesis, Universidad de Chile, 1998.

[Gaj01] A. Gajardo. Dependence of the behavior of the dynamical system Langton's ant on the network topology. ?, Universidad de Chile et École normale sup'erieure de Lyon, 2001.

[Ga193] D. Gale. The industrious ant. Math. Intelligencer, 15:54-58, 1993.

[GBCB99] P. Grosfi ls, J. P. Boon, E. G. D. Cohen, and L. A. Bunimovich. Propagation and organization in a lattice random media. J. Stat. Physics, 97(3-4):575-608, 1999.

[GG01] A. Gajardo and E. Goles. Universal cellular automaton over a hexagonal tiling with 3 states. International Journal of Algebra and Computation, 11(3):335-354, 2001.

[GMG01a] A. Gajardo, A. Moreira, and E. Goles. Complexity of langton's an. to appear in Discrete Applied Mathematics, ?(?):?, 2001.

[GMG01b] A. Gajardo, A. Moreira, and E. Goles. Generalized langton's ant: Dynamical behavior and complexity. In A. Ferreira and H. Reichel, editors, STACS 2001, volume 2010 of Lecture Notes in Computer Science, pages 259-270, 2001.

[GPST95] D. Gale, J. Propp, S. Sutherland, and S. Troubetzkoy. Further travels with my ant. Math. Intelligencer, 17(3):48-56, 1995.

[KC91] X. P. Kong and E. G. D. Cohen. Diffusion and propagation in a triangular lorentz lattice gas cellular automata. J. Stat. Physics, 62:737, 1991.

[Kit98] B. Kitchens. Symbolic Dynamics. Springer, 1998. 
[Kle99] J. M. Kleinberg. Authoritative sources in a hyperlinked enviroment. Journal of the ACM, 46(5):604-632, 1999.

[K9̊7] P. Kůrka. On topological dynamics of turing machines. Teoret. Comput. Sci., 174(1-2):203216, 1997.

[Lan86] C. G. Langton. Studing artifi tial life with cellular automata. Physica D, 22:120-149, 1986.

[LS77] R. C. Lyndon and P. E. Schupp. Combinatorial Group Theory. Springer-Verlag, 1977.

[MGG01] A. Moreira, A. Gajardo, and E. Goles. Dynamical behavior and complexity of langton's ant. Complexity, 6(4):46-52, 2001.

[PR00] C. Papazian and E. R'emila. Some properties of hyperbolic discrete planes. In G. Borgefors, I. Nyström, and G. Sanniti di Baja, editors, DGCI, volume 1953 of Lecture Notes in Computer Science. Springer, 2000.

[Qua99] A. Quas. Infi nite paths in a lorentz lattice gas model. Probab. Theory Rel, 114(2):229-244, 1999.

[Tro97] S. Troubetzkoy. Lewis-parker lecture 1997 the ant. Alabama J. Math., 21(2):3-15, 1997.

[Van] http://www.dim.uchile.cl/ agajardo/langton.

[Wan95] F. Wang. PhD thesis, The Rockefeller University, 1995. 